\title{
Grand Challenges for Artificial Intelligence in Molecular Medicine
}

\author{
Frank Emmert-Streib ${ }^{1,2 *}$ \\ ${ }^{1}$ Predictive Society and Data Analytics Lab, Faculty of Information Technolgy and Communication Sciences, Tampere University, \\ Tampere, Finland, ${ }^{2}$ Institute of Biosciences and Medical Technology, Tampere, Finland
}

Keywords: artificial intelligence, molecular medicine, machine learning, biomedicine, omics, data science, health data science, biomedical data science

\section{INTRODUCTION}

In recent years, novel methods from artificial intelligence (AI) and machine learning (ML) commonly referred to as data science (DS) enabled many advances in data-driven fields including computational biology, bioinformatics, network medicine, precision medicine and systems medicine (He et al., 2019; Rajkomar et al., 2019; Zou et al., 2019). Given the continuation of technological innovations that will further lead to new high-throughput measurements on all molecular levels, it can be expected that the importance of AI and ML for medicine and biomedicine will even increase in the future (Obermeyer and Emanuel, 2016; EmmertStreib, 2021). For this reason, a scientific forum is needed for nurturing methodological developments and practical applications of $\mathrm{AI}, \mathrm{ML}$ and general DS in molecular medicine allowing the community to disseminate and discuss recent results.

The Bioinformatics and AI Specialty Section aims to provide such a forum for publishing articles about the analysis of all types of Omics, clinical and health data for enhancing our understanding of molecular medicine. The emphasize is on either the application or the development of data-driven methods for diagnostic, prognostic, predictive or exploratory studies based on methods from AI or ML.

In Figure 1, we show an overview of the iterative process of scientific discovery utilizing artificial

Edited and reviewed by: Masaru Katoh, National Cancer Centre, Japan

*Correspondence: Frank Emmert-Streib v@bio-complexity.com

Specialty section:

This article was submitted to Bioinformatics and Artificial Intelligence for Molecular Medicine, a section of the journal Frontiers in Molecular Medicine

Received: 01 July 2021

Accepted: 08 July 2021

Published: 22 July 2021

Citation:

Emmert-Streib F (2021) Grand Challenges for Artificial Intelligence in

Molecular Medicine.

Front. Mol. Med. 1:734659. doi: $10.3389 /$ fmmed.2021.734659 intelligence and machine learning to enhance our knowledge about molecular medicine. In the following, we discuss several of these topics that are in our opinion of particular relevance for the development of $\mathrm{AI}$ and ML in molecular medicine.

\section{DATA: UTILIZATION AND INTEGRATION}

In (Feldman et al., 2012), "data" have been called "fuel” because it is like oil for scientific discoveries. For this reason, it is not surprising that we start by discussing the importance of data for molecular medicine. In general, all methods from data science, regardless if they have their origin in artificial intelligence, machine learning or statistics, are based on data (Emmert-Streib and Dehmer, 2019). In other words, a method alone is not capable of contributing anything of meaning for molecular medicine but the combination with data is required.

Nowadays there are many big data resources available that can be utilized for developing and testing methods. Prominent examples thereof are The Cancer Genome Atlas Research Network (TCGA) (The Cancer Genome Atlas Research Network, 2008), Gene ontology (GO) (Ashburner et al., 2000), Gene Expression Omnibus (GEO) (Edgar et al., 2002) or Library of Integrated Network-based Cellular Signatures (LINCS) (Koleti et al., 2017). Interestingly, the idea that such data can also be used for making novel discoveries about the molecular understanding of disorders is so far largely underexplored.

A common problem encountered is how the diverse and often heterogeneous data can be integrated in a meaningful and sound way (Zitnik et al., 2019). Traditionally, one tried to accomplish this by the 


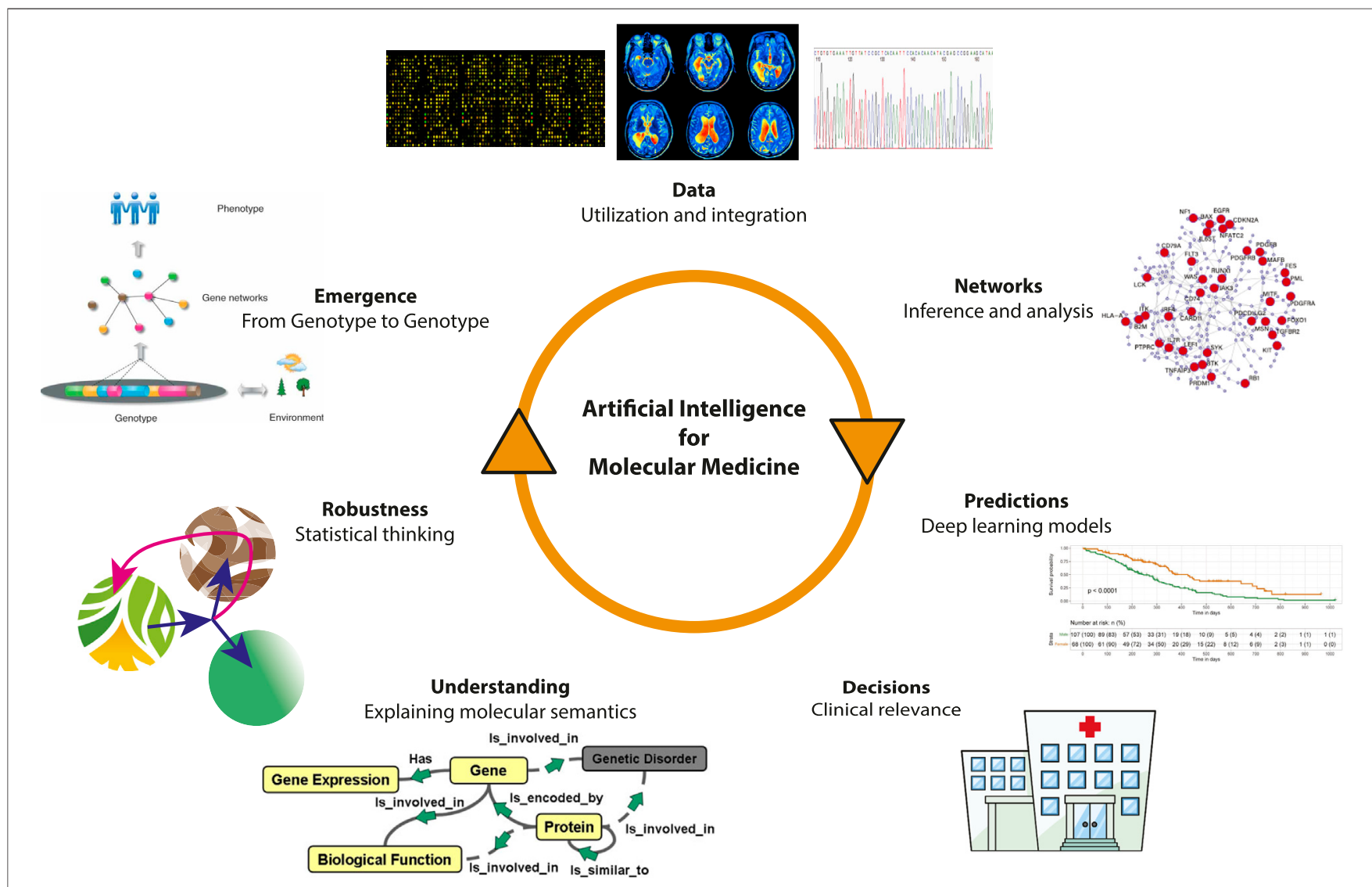

FIGURE 1 | The iterative process of scientific discovery utilizing artificial intelligence and machine learning to enhance our knowledge about molecular medicine.

normalization of data with the hope that this allows the pooling of data, i.e., two or more data sets can be combined, from different sources. While this approach is applicable in certain situations it does not offer a generic solution. Instead, a conceptual approach that could be of great practical relevance in this context is provided by transfer learning (Pan and Yang, 2009). The basic idea of transfer learning is to utilize data from two different domains and to use both for learning a so called target task. Importantly, the underlying feature spaces of both domains can be different. Hence, this framework allows to utilize data from different domains without actually combining them. For instance, data from DNA microarrays can be used to improve tasks for RNA-seq data or even to utilize imaging data, e.g., from $\mathrm{X}$-Rays or fMRIs, or text data from electronic health records (eHR) for the same target task. Other machine learning paradigms that could be of relevance are multi-task learning or semi-supervised learning (Chapelle et al., 2006; Zhang and Yang, 2018).

\section{NETWORKS: INFERENCE AND ANALYSIS}

Another type of approach that is of crucial relevance for molecular medicine is network-based approaches (Vidal, 2009). Specifically, there have been many studies inferring various types of gene regulatory networks (GRNs), including transcription regulation networks, protein interaction networks, metabolic networks or signalling networks (Emmert-Streib and Dehmer, 2018). Each of these provide useful information about molecular interactions on the cellular level (Emmert-Streib et al., 2014). However, in order to obtain a full systems biology understanding an integration of such networks is needed. Hence, multi-scale network studies are needed to provide us with comprehensive blue-prints about the hierarchical molecular organization pattern (Yu and Gerstein, 2006; Ravasz, 2009).

A field that is dedicated for utilizing such approaches is network medicine (Barabási, 2007). A particular example for the utility of networks is to study the relations between disorders and genes (Goh et al., 2007; Emmert-Streib et al., 2013). Importantly, instead of focusing on individual disorders or genes at a time, network medicine aims at providing insights into the intricate interrelations among all such entities. This allows not only the exploitation of common biological processes or pathways but also to make predictions, e.g., about the drug repurposing (AY et al., 2007; Pushpakom et al., 2019). Hence, networks provide efficient means for studying basic molecular biological questions of disorders and pharmacogenomic problems to gain insights into treatment options for patients. 


\section{PREDICTIONS: DEEP LEARNING MODELS}

A good example to show that machine learning and artificial intelligence are dynamical fields with constant innovations is deep learning (LeCun et al., 2015). Methods of this type came to the awareness of the general community around 2012 and have since then contributed to enhance our understanding in many domains. One particular reason contributing to the success of deep learning methods is the flexibility they offer for building neural networks of different tasks. As a result, nowadays a large number of network architectures is known, e.g., Convolutional Neural Networks (CNN), Long ShortTerm Memory networks (LSTM) or Deep Belief Networks, that have been applied in a large variety of application domains (Schmidhuber, 2015; Emmert-Streib et al., 2020a).

One particular deep learning model for the analysis of text data that received considerable attention is BERT (Bidirectional Encoder Representations from Transformers) (Devlin et al., 2019). BERT is an autoencoding language model trained using stacked encoder blocks from transformers with a masked language modeling (MLM) to learn word-embeddings bidirectionally. Part of the success of this model is its flexibility to be utilized for a number of different prediction tasks, including named entity recognition, question answering and relation detection (Perera et al., 2020). Hence, this model is of great relevance for analyzing, e.g., electronic Health Records (eHR) from hospitals (Lee et al., 2019; Li et al., 2019).

\section{DECISIONS: CLINICAL RELEVANCE}

Of particular practical relevance for molecular medicine are studies investigating diagnostic, predictive, prognostic or therapeutic signatures of biomarkers. The reason for this it that such studies have the potential to inform clinical decision making by influencing the diagnosis or treatment of patients in profound ways. The surge of genomics data provides ample opportunities for such studies and one key issue of these is feature selection. Specifically, while the number of molecular entities, e.g., about genes or mRNAs, is in the tens of thousands, interpretable models aim to limit this number to the smallest possible number.

Another interesting topic in this context is the utility of network biomarkers. In contrast to traditional approaches that are based on, e.g., sets of genes or proteins, network biomarkers utilize structural features from gene regulatory networks (Chen et al., 2012; Zeng et al., 2013). This converts a structureless set of genes (sometimes called gene bag) into a complex entity conveying more predictive and interpretable information. As a side-note we would like to mention that this could be also beneficial for the visualization of results and the doctor-patient communication in order to explain therapeutic measures.

\section{UNDERSTANDING: EXPLAINING MOLECULAR SEMANTICS}

A common goal of all above approaches is to enhance our understanding of the molecular bases of disorders. In order to see that this is a non-trivial endeavour let's discuss some examples. Deep learning models have been criticized for being black-box models (Adadi and Berrada, 2018). That means such models are good for making predictions but defy a straightforward interpretation making the models non-explainable (Emmert-Streib et al., 2020b). This is particularly problematic in a medical context involving humans because this utimately means that clinical decisions, e.g., based on the analysis of personal genomics data, cannot be explained to the patient.

Another example is given by biomarkers. In general, biomarkers are used for diagnostic, prognostic, predictive or therapeutic purposes to make decisions about the care of a patient (Califf, 2018). It is widely believed that aside from this clinical utility based on the predictive capabilities of such signatures, biomarkers are also offering insights into the molecular functioning of biological processes and their causal involvement in disorders (Van De Vijver et al., 2002; Cuzick et al., 2011). However, for prognostic signatures of breast cancer it has been demonstrated that this is not the case (Venet et al., 2011; Manjang et al., 2021). This implies that also the prognostic signatures are black-box models with sensible predictions of breast cancer outcome but no value for revealing causal connections. Hence, such models have a predictive utility, e.g., for applications in the clinical practice but no biological utility for enhancing our understanding of breast cancer biology. If similar results are observed for other cancer types or different disorders remains to be seen.

From these examples one can see that establishing a good prediction model does not impliy that we also obtain immediately an understanding of the molecular semantics offered by disorders. Hence, ideally, causal prediction models are required that provide prediction capabilities along with an interpretable structure for giving causal explanations of molecular activities (Holzinger et al., 2019). In case such ideal models are unachievable one needs measures for quantifying these deficiencies.

\section{ROBUSTNESS: STATISTICAL THINKING}

An aspect that does not receive enough appreciation is the fact that any type of the analysis of data from molecular medicine requires statistical considerations. That means even modern developments in $\mathrm{AI}$ and $\mathrm{ML}$ do not make a statistical understanding obsolete but are built upon it. This includes, for instance, ensuring the reproducability of studies (Peng, 2011; Begley and Ioannidis, 2015), multiple testing corrections of hypotheses (Noble, 2009) or the regularization of regression models (Tibshirani, 1996). Of particular interest are studies that clarify the understanding of problems of widely used methods or approaches (Ioannidis, 2005; Tripathi et al., 2013; Wasserstein and Lazar, 2016). Hence, investigations that enhance our understanding of molecular medicine by applying any form of statistical thinking are welcome to advance bioinformatics because only such approaches lead to the robustness of findings that are of biological and clinical significance (Vingron, 2001). 


\section{EMERGENCE: FROM GENOTYPE TO PHENOTYPE}

Finally, we would like to emphasize that molecular medicine aims to study the connection between genotype and phenotype (Ginsburg and Willard, 2009; Collins and Varmus, 2015). That means, while aberrant molecular processes give rise to various forms of disorders, those molecular processes should not be studied in isolation but their phenotypic consequences need to be systematically documented. However, this requires to bridge from genotype to phenotype (Noble, 2008a; Gjuvsland et al., 2013; Ritchie et al., 2015). For practical approaches 'networks' have been suggested to capture relevant information as an intermediate layer (Emmert-Streib and Glazko, 2011; Carter et al., 2013; Kim and Przytycka, 2013), however, further instigations are needed, e.g., to merge such approaches with predictive models.

On a theoretical note, we would like to highlight that the above problem is actually severe because it requires an understanding of emergence (Noble, 2008b; Pigliucci, 2010). Hence, reductionist approaches are prone to fail in molecular medicine which possesses major challenges for conceptual approaches provided by AI or ML to overcome such limitations (Mazzocchi, 2012). Hence, even personalized medicine or precision medicine depend on our theoretical understanding of the biological complexity

\section{REFERENCES}

Adadi, A., and Berrada, M. (2018). Peeking inside the Black-Box: A Survey on Explainable Artificial Intelligence (XAI). IEEE Access. 6, 52138-52160. doi:10.1109/access.2018.2870052

Ashburner, M., Ball, C. A., Blake, J. A., Botstein, D., Butler, H., Michael Cherry, J., et al. (2000). Gene Ontology: Tool for the Unification of Biology. The Gene Ontology Consortium. Nat. Genet. 25, 25-29. doi:10.1038/75556

Ay, M., Goh, K. I., Cusick, M. E., Barabasi, A. L., and Vidal, M. (2007). Drug-target Network. Nat. Biotechnol. 25, 1119-1127. doi:10.1038/nbt1338

Barabási, A.-L. (2007). Network Medicine - from Obesity to the "Diseasome". N. Engl. J. Med. 357, 404-407. doi:10.1056/nejme078114

Begley, C. G., and Ioannidis, J. P. (2015). Reproducibility in Science: Improving the Standard for Basic and Preclinical Research. Circ. Res. 116, 116-126. doi:10.1161/circresaha.114.303819

Califf, R. M. (2018). Biomarker Definitions and Their Applications. Exp. Biol. Med. 243, 213-221. doi:10.1177/1535370217750088

Carter, H., Hofree, M., and Ideker, T. (2013). Genotype to Phenotype via Network Analysis. Curr. Opin. Genet. Dev. 23, 611-621. doi:10.1016/j.gde.2013.10.003

Chapelle, O., Schölkopf, B., and Zien, A. (2006). Semi-Supervised Learning. Adaptive Computation and Machine Learning. The MIT Press.

Chen, L., Liu, R., Liu, Z.-P., Li, M., and Aihara, K. (2012). Detecting Early-Warning Signals for Sudden Deterioration of Complex Diseases by Dynamical Network Biomarkers. Scientific Rep. 2, 1-8. doi:10.1038/srep00342

Collins, F. S., and Varmus, H. (2015). A New Initiative on Precision Medicine. New Engl. J. Med. 372, 793-795. doi:10.1056/nejmp1500523

Cuzick, J., Swanson, G. P., Fisher, G., Brothman, A. R., Berney, D. M., Reid, J. E., et al. (2011). Prognostic Value of an Rna Expression Signature Derived from Cell Cycle Proliferation Genes in Patients with Prostate Cancer: a Retrospective Study. Lancet Oncol. 12, 245-255. doi:10.1016/ s1470-2045(10)70295-3

Devlin, J., Chang, M.-W., Lee, K., and Toutanova, K. (2019). "Bert: Pre-training of Deep Bidirectional Transformers for Language Understanding," in Proceedings of emergent features arising from the transition between the genotype to the phenotype.

\section{CONCLUSION}

The Bioinformatics and AI Specialty Section of Frontiers in Molecular Medicine will provide a venue for world-class interdisciplinary research addressing the above, and many more challenges arising in the future. In order to provide a forum for the exchange of ideas and growth of innovations for a multi-disciplinary research community, the journal does not only publish Original Research and Review articles but a number of additional paper types. For instance, the journal welcomes submissions for the following article types: Hypothesis and Theory, Perspective, Opinion and General Commentary. This will allow to express the perspectives and opinions of the community and to discuss recent developments critically. Furthermore, the journal publishes also Technology and Code articles which present 1) new software, 2) new applications of software or 3) implementations of existing algorithms under novel settings.

\section{AUTHOR CONTRIBUTIONS}

The author confirms being the sole contributor of this work and has approved it for publication.

of the 2019 Conference of the North American Chapter of the Association for Computational Linguistics: Human Language Technologies, Volume 1 (Long and Short Papers), Minneapolis, Minnesota. 4171-4186.

Edgar, R., Domrachev, M., and Lash, A. E. (2002). Gene Expression Omnibus: NCBI Gene Expression and Hybridization Array Data Repository. Nucleic Acids Res. 30, 207-210. doi:10.1093/nar/30.1.207

Emmert-Streib, F., and Dehmer, M. (2018). Inference of Genome-Scale Gene Regulatory Networks: Are There Differences in Biological and Clinical Validations? Mach. Learn. Knowl. Extr. 1, 138-148. doi:10.3390/make1010008

Emmert-Streib, F., and Dehmer, M. (2019). Defining Data Science by a DataDriven Quantification of the Community. Mach. Learn. Knowl. Extr. 1, 235-251. doi:10.3390/make1030058

Emmert-Streib, F., Dehmer, M., and Haibe-Kains, B. (2014). Gene Regulatory Networks and Their Applications: Understanding Biological and Medical Problems in Terms of Networks. Front. Cel Dev. Biol. 2, 38. doi:10.3389/ fcell.2014.00038

Emmert-Streib, F. (2021). From the Digital Data Revolution toward a Digital Society: Pervasiveness of Artificial Intelligence. Mach. Learn. Knowl. Extr. 3, 284-298. doi:10.3390/make3010014

Emmert-Streib, F., and Glazko, G. (2011). Network Biology: A Direct Approach to Study Biological Function. Wiley Interdiscip. Rev. Syst. Biol. Med. 3, 379-391. doi:10.1002/wsbm.134

Emmert-Streib, F., Tripathi, S., de Matos Simoes, R., Hawwa, A., and Dehmer, M. (2013). The Human Disease Network: Opportunities for Classification, Diagnosis and Prediction of Disorders and Disease Genes. Syst. Biomed. 1, 1-8. doi:10.4161/sysb.22816

Emmert-Streib, F., Yang, Z., Feng, H., Tripathi, S., and Dehmer, M. (2020a). An Introductory Review of Deep Learning for Prediction Models with Big Data. Front. Artif. Intelligence 3, 4. doi:10.3389/frai.2020.00004

Emmert-Streib, F., Yli-Harja, O., and Dehmer, M. (2020b). Explainable Artificial Intelligence and Machine Learning: A Reality Rooted Perspective. WIREs Data Mining Knowledge Discov. 10, e1368. doi:10.1002/widm.1368

Feldman, B., Martin, E. M., and Skotnes, T. (2012). Big Data in Healthcare Hype and hope. Dr. Bonnie. 360, 122-125. doi:10.1109/TKDE.2009.191 
Ginsburg, G. S., and Willard, H. F. (2009). Genomic and Personalized Medicine: Foundations and Applications. Translational Res. 154, 277-287. doi:10.1016/ j.trsl.2009.09.005

Gjuvsland, A. B., Vik, J. O., Beard, D. A., Hunter, P. J., and Omholt, S. W. (2013). Bridging the Genotype-Phenotype gap: what Does it Take? J. Physiol. 591, 2055-2066. doi:10.1113/jphysiol.2012.248864

Goh, K.-I., Cusick, M. E., Valle, D., Childs, B., Vidal, M., and Barabasi, A. (2007). The Human Disease Network. Proc. Natl. Acad. Sci. 104, 8685-8690. doi:10.1073/pnas.0701361104

He, J., Baxter, S. L., Xu, J., Xu, J., Zhou, X., and Zhang, K. (2019). The Practical Implementation of Artificial Intelligence Technologies in Medicine. Nat. Med. 25, 30-36. doi:10.1038/s41591-018-0307-0

Holzinger, A., Langs, G., Denk, H., Zatloukal, K., and Müller, H. (2019). Causability and Explainability of Artificial Intelligence in Medicine. Wiley Interdiscip. Rev. Data Mining Knowledge Discov. 9, e1312. doi:10.1002/widm.1312

Ioannidis, J. P. A. (2005). Why Most Published Research Findings Are False. Plos Med. (New York: Springer) 2, e124. doi:10.1371/journal.pmed.0020124

Kim, Y.-A., and Przytycka, T. M. (2013). Bridging the gap between Genotype and Phenotype via Network Approaches. Front. Genet. 3, 227. doi:10.3389/ fgene.2012.00227

Koleti, A., Terryn, R., Stathias, V., Chung, C., Cooper, D. J., Turner, J. P., et al. (2017). Data portal for the Library of Integrated Network-Based Cellular Signatures (Lincs) Program: Integrated Access to Diverse Large-Scale Cellular Perturbation Response Data. Nucleic Acids Res. 46, D558-D566. doi:10.1093/nar/gkx1063

LeCun, Y., Bengio, Y., and Hinton, G. (2015). Deep Learning. Nature 521, 436. doi:10.1038/nature14539

Lee, J., Yoon, W., Kim, S., Kim, D., Kim, S., So, C. H., et al. (2019). BioBERT: a Pretrained Biomedical Language Representation Model for Biomedical Text Mining. Bioinformatics 36. doi:10.1093/bioinformatics/btz682

Li, F., Jin, Y., Liu, W., Rawat, B. P. S., Cai, P., and Yu, H. (2019). Fine-tuning Bidirectional Encoder Representations from Transformers (Bert)-based Models on Large-Scale Electronic Health Record Notes: an Empirical Study. JMIR Med. Inform. 7, e14830. doi:10.2196/14830

Manjang, K., Tripathi, S., Yli-Harja, O., Dehmer, M., Glazko, G., and EmmertStreib, F. (2021). Prognostic Gene Expression Signatures of Breast Cancer Are Lacking a Sensible Biological Meaning. Scientific Rep. 11, 1-18. doi:10.1038/ s41598-020-79375-y

Mazzocchi, F. (2012). Complexity and the Reductionism-Holism Debate in Systems Biology. Wiley Interdiscip. Rev. Syst. Biol. Med. 4, 413-427. doi:10.1002/wsbm.1181

Noble, D. (2008a). Genes and Causation. Phil. Trans. R. Soc. A. 366, 3001-3015. doi:10.1098/rsta.2008.0086

Noble, D. (2008b). The Music of Life: Biology beyond Genes. Oxford University Press.

Noble, W. S. (2009). How Does Multiple Testing Correction Work?. Nat. Biotechnol. 27, 1135. doi:10.1038/nbt1209-1135

Obermeyer, Z., and Emanuel, E. J. (2016). Predicting the Future-Big Data, Machine Learning, and Clinical Medicine. New Engl. J. Med. 375, 1216. doi:10.1056/nejmp1606181

Pan, S. J., and Yang, Q. (2009). A Survey on Transfer Learning. IEEE Trans. knowledge Data Eng. 22, 1345-1359.

Peng, R. D. (2011). Reproducible Research in Computational Science. Science 334, 1226-1227. doi:10.1126/science.1213847

Perera, N., Dehmer, M., and Emmert-Streib, F. (2020). Named Entity Recognition and Relation Detection for Biomedical Information Extraction. Front. Cel Dev. Biol. 8, 673. doi:10.3389/fcell.2020.00673

Pigliucci, M. (2010). Genotype-phenotype Mapping and the End of the 'genes as Blueprint'metaphor. Philos. Trans. R. Soc. B: Biol. Sci. 365, 557-566. doi:10.1098/rstb.2009.0241
Pushpakom, S., Iorio, F., Eyers, P. A., Escott, K. J., Hopper, S., Wells, A., et al. (2019). Drug Repurposing: Progress, Challenges and Recommendations. Nat. Rev. Drug Discov. 18, 41-58. doi:10.1038/nrd.2018.168

Rajkomar, A., Dean, J., and Kohane, I. (2019). Machine Learning in Medicine. New Engl. J. Med. 380, 1347-1358. doi:10.1056/nejmra1814259

Ravasz, E. (2009). "Detecting Hierarchical Modularity in Biological Networks," in Computational Systems Biology. Springer, 1-16. doi:10.1007/978-1-59745-243-4_7

Ritchie, M. D., Holzinger, E. R., Li, R., Pendergrass, S. A., and Kim, D. (2015). Methods of Integrating Data to Uncover Genotype-Phenotype Interactions. Nat. Rev. Genet. 16, 85-97. doi:10.1038/nrg3868

Schmidhuber, J. (2015). Deep Learning in Neural Networks: An Overview. Neural networks. 61, 85-117. doi:10.1016/j.neunet.2014.09.003

The Cancer Genome Atlas Research Network (2008). Comprehensive Genomic Characterization Defines Human Glioblastoma Genes and Core Pathways. Nature 455, 1061-1068. doi:10.1038/nature07385

Tibshirani, R. (1996). Regression Shrinkage and Selection via the Lasso. J. R. Stat. Soc. Ser. B. 58, 267-288. doi:10.1111/j.2517-6161.1996.tb02080.x

Tripathi, S., Glazko, G., and Emmert-Streib, F. (2013). Ensuring the Statistical Soundness of Competitive Gene Set Approaches: Gene Filtering and GenomeScale Coverage Are Essential. Nucleic Acids Res. 6, e53354. doi:10.1093/nar/ gkt054

Van De Vijver, M. J., He, Y. D., Van't Veer, L. J., Dai, H., Hart, A. A., Voskuil, D. W., et al. (2002). A Gene-Expression Signature as a Predictor of Survival in Breast Cancer. New Engl. J. Med. 347, 1999-2009. doi:10.1056/nejmoa021967

Venet, D., Dumont, J. E., and Detours, V. (2011). Most Random Gene Expression Signatures Are Significantly Associated with Breast Cancer Outcome. PLoS Comput. Biol. 7, e1002240. doi:10.1371/journal.pcbi.1002240

Vidal, M. (2009). A Unifying View of 21st century Systems Biology. FEBS Lett. 583, 3891-3894. doi:10.1016/j.febslet.2009.11.024

Vingron, M. (2001). [Dataset], Bioinformatics Needs to Adopt Statistical Thinking Bioinformatics 17 (5), 389-390. doi:10.1093/bioinformatics/17.5.389

Wasserstein, R. L., and Lazar, N. A. (2016). The ASA's Statement on P-Values: Context, Process, and Purpose. The Am.Stat. 70, 129-133. doi:10.1080/ 00031305.2016.1154108

Yu, H., and Gerstein, M. (2006). Genomic Analysis of the Hierarchical Structure of Regulatory Networks. Proc. Natl. Acad. Sci. USA. 103, 14724-14731. doi:10.1073/pnas.0508637103

Zeng, T., Sun, S.-y., Wang, Y., Zhu, H., and Chen, L. (2013). Network Biomarkers Reveal Dysfunctional Gene Regulations during Disease Progression. FEBS J. 280, 5682-5695. doi:10.1111/febs. 12536

Zhang, Y., and Yang, Q. (2018). An Overview of Multi-Task Learning. Natl. Sci. Rev. 5, 30-43. doi:10.1093/nsr/nwx105

Zitnik, M., Nguyen, F., Wang, B., Leskovec, J., Goldenberg, A., and Hoffman, M. M. (2019). Machine Learning for Integrating Data in Biology and Medicine: Principles, Practice, and Opportunities. Inf. Fusion 50, 71-91. doi:10.1016/ j.inffus.2018.09.012

Zou, J., Huss, M., Abid, A., Mohammadi, P., Torkamani, A., and Telenti, A. (2019). A Primer on Deep Learning in Genomics. Nat. Genet. 51, 12-18. doi:10.1038/ s41588-018-0295-5

Conflict of Interest: The author declares that the research was conducted in the absence of any commercial or financial relationships that could be construed as a potential conflict of interest.

Copyright (C) 2021 Emmert-Streib. This is an open-access article distributed under the terms of the Creative Commons Attribution License (CC BY). The use, distribution or reproduction in other forums is permitted, provided the original author(s) and the copyright owner(s) are credited and that the original publication in this journal is cited, in accordance with accepted academic practice. No use, distribution or reproduction is permitted which does not comply with these terms. 